Check for updates

Cite this: RSC Adv., 2018, 8, 7892

\section{A microwave synthesized mesoporous carbon sponge as an efficient adsorbent for $\mathrm{Cr}(\mathrm{VI})$ removal $\uparrow$}

\begin{abstract}
Yan-Jun Liu, ${ }^{a}$ Shan Liu, ${ }^{a}$ Zhi-Wen Li, ${ }^{a}$ Ming-Guo Ma (D)*ab and Bo Wang ${ }^{a}$
Mesoporous carbon materials have recently attracted immense research interest because of their potential application in water purification fields. Herein, we report the synthesis of a mesoporous carbon sponge (MCS) from a supramolecular microcrystalline cellulose-polymer system triggered by microwaveassisted treatment. Benefiting from the three-dimensional (3D) interconnected mesopores and an evenly distributed ball-like protuberance on the inner surfaces of the macropores, the MCS exhibited a high adsorption capacity $\left(93.96 \mathrm{mg} \mathrm{g}^{-1}\right.$ ) for fast $\mathrm{Cr}(\mathrm{vI})$ removal within $5 \mathrm{~min}$. Additionally, the MCS can be regenerated and reused after the adsorption-desorption process, and maintained an adsorption capacity of $\sim 86 \%$ after 10 cycles. The high adsorption capacity, significantly reduced treatment time, and reusability make the MCS promising for the purification of wastewater on a large scale.
\end{abstract}

Received 2nd January 2018

Accepted 2nd February 2018

DOI: $10.1039 / \mathrm{c} 8 \mathrm{ra} 00012 \mathrm{c}$

rsc.li/rsc-advances variants, including activated carbon (AC), ${ }^{\mathbf{1 4}}$ magnetic carbon nanocomposites (MCNs), ${ }^{15-18}$ carbon nanofibers (CNFs), ${ }^{19}$ carbon nanotubes (CNTs), ${ }^{\mathbf{2 0 , 2 1}}$ carbon membranes (CMs), carbon sponge (CS), ${ }^{2-24}$ and carbon aerogel (CA) ${ }^{25-29}$ Until now, some successful strategies such as the thermal decomposition, ${ }^{12}$ electrochemical deposition, ${ }^{\mathbf{3 0 , 3 1}}$ layer-by-layer technique, ${ }^{20,32}$ self-assembly, ${ }^{33}$ and synergistically assembly ${ }^{29}$ were employed for the synthesis of advanced mesoporous carbon materials. Among them, microwave-assisted thermal treatment could not only selectively couple with the intermediate in a multiphase reaction system but also overcome the high activation energies for product formation. ${ }^{34}$ Moreover, the microwave heating reduces the overall thermal gradients in the reaction and thus yields more uniform products. ${ }^{35}$ Hence, microwave-assisted thermal treatment, as an economic, more efficient, and green method, is an alternative technology for preparing mesoporous carbon materials. However, as far as we know, the preparation of MCS from a supramolecular microcrystalline cellulose-polymer system via synergistically assembly triggered by microwave-assisted treatment has not been reported yet.

In this work, an environmentally friendly strategy was applied to prepare mesoporous carbon sponge (MCS) with three-dimensional (3D) interconnected structures by the microwave-assisted method using microcrystalline cellulose (MCC), poly(vinyl alcohol) (PVA), and poly(vinyl pyrrolidone) (PVP) (Fig. 1). The molecular chains of PVA, PVP, and MCC served as carbon sponge precursors, which undergo synergistically assembly upon the simultaneous covalent polymerization and physical interaction triggered by microwave-assisted heating/annealing treatments. The microwave-assisted synthetic process is simple and time-saving, and thus the product can be easily manufactured in a large-scale.
${ }^{a}$ Engineering Research Center of Forestry Biomass Materials and Bioenergy, Beijing Key Laboratory of Lignocellulosic Chemistry, College of Materials Science and Technology, Beijing Forestry University, Beijing 100083, PR China. E-mail:mg_ma@bjfu.edu.cn

${ }^{b}$ Key Laboratory of Pulp and Paper Science \& Technology of Ministry of Education/ Shandong Province, Qilu University of Technology, Jinan 250353, PR China

$\dagger$ Electronic supplementary information (ESI) available: UV-Vis absorption of the $\mathrm{Cr}(\mathrm{vI})$ solution treated with different carbon sponges and video of the process of the carbon sponges for $\mathrm{Cr}(\mathrm{vI})$ removal. See DOI: $10.1039 / \mathrm{c} 8 \mathrm{ra00012c}$ 


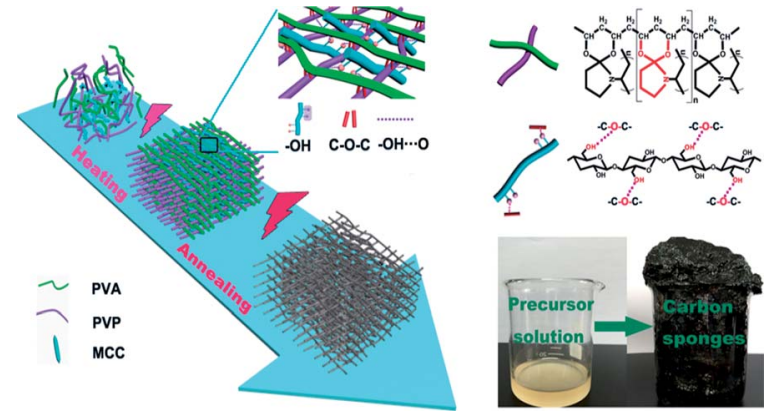

Fig. 1 Schematic illustration of the formation of carbon sponge (PPMC) (inset: photo of the carbon sponge).

Subsequently, we focused our efforts on studying $\mathrm{Cr}(\mathrm{vI})$ removal through the MCS. The as-prepared MCS exhibited exceptionally high adsorption capacity of $93.96 \mathrm{mg} \mathrm{g}^{-1}$ and fast $\mathrm{Cr}(\mathrm{vI})$ removal from wastewater only within $5 \mathrm{~min}$.

\section{Experimental}

\section{Materials}

All chemicals used here were of analytical grade and used as received without further purification. All experiments were operated under an ambient atmosphere. Microcrystalline cellulose (MCC, molecular weight of 34 843-38 894 degree of polymerization (DP, DP $=215-240)$ ) of a commercial reagent was purchased from Sinopharm Group Chemical Reagent Co., Ltd. Poly(vinyl alcohol) (PVA, $\sim 88 \%$ hydrolyzed, DP $=1750 \pm$ 50) was purchased from Sinopharm Chemical Reagent Co., Ltd. and poly(vinyl pyrrolidone) (PVP, K-30) was purchased from Xilong Chemical. Potassium dichromate $\left(\mathrm{K}_{2} \mathrm{Cr}_{2} \mathrm{O}_{7}\right)$, sulfuric acid $(98 \%)$, sodium hydroxide $(\mathrm{NaOH})$, and sodium chloride $(\mathrm{NaCl})$ were purchased from Beijing Chemical Works.

\section{Preparation of carbon sponges}

For the preparation of the MCS, typically, solution A was prepared by dissolving $10 \mathrm{~g}$ PVA in $115 \mathrm{~mL}$ distilled water, under vigorous stirring $(800 \mathrm{rpm})$ at $85{ }^{\circ} \mathrm{C}$ for $15 \mathrm{~min}$. And solution B was prepared by dissolving $7.5 \mathrm{~g}$ PVP in $42.5 \mathrm{~mL}$ distilled water under ultrasonic wave $(100 \mathrm{~W})$ for $10 \mathrm{~min}$. Two different strategies were used to prepare the MCS: (a) the solution $\mathrm{A}$ and $\mathrm{B}$ were mixed uniformity of their volume ratio of $7: 3$, and the sulfuric acid $(\omega=10 \mathrm{wt} \%, 1.5 \%$ of the reaction mixture) was added to the reaction mixture under vigorous stirring, obtaining the precursor solution. The precursor solution was then poured into a glass beaker $(100 \mathrm{~mL})$ for immediate exposure to microwave energy at $300 \mathrm{~W}$ using UWave-1000 (Shanghai Sineo) in a high purity $\mathrm{N}_{2}$ atmosphere for $5 \mathrm{~min}$, and then microwave-assisted annealing at $900 \mathrm{~W}$ for 10 min, obtaining the MCS and the resulting product was named PP-C. (b) The other MCS was fabricated by the same strategy as in (a), and the only difference is that the precursor solution consist of solution $\mathrm{A}$ and $\mathrm{B}$, the sulfuric acid, and MCC (1.5\% of the reaction mixture). The resulting product was named PPM-C (Table S1†).

\section{Characterization}

The morphology of the samples was characterized using scanning electron microscopy (SEM, SU8010) with a field emission gun, operated at an accelerating voltage of $10.0 \mathrm{kV}$. The energy-dispersive X-ray spectra (EDS) attached to the scanning electron microscopy (ZEISS EVO18) was used to analyze the composition of sample (scanning area: $4 \times 5 \mu^{2}$ ), operated at an accelerating voltage of $15.0 \mathrm{kV}$ (takeoff angle $=$ $35.0^{\circ}$ elapsed livetime $=9.2$ ). Fourier transform infrared (FTIR) spectroscopy was obtained on Thermo Scientific Nicolet iN10 FTIR Microscope (Thermo Nicolet Corporation, Madison, WI, USA), which was equipped with a liquid nitrogen cooled MCT detector. The X-ray photoelectron spectroscopy (XPS) measurements were performed on Thermo Scientific Escalab 250Xi XPS system (Thermo Fisher Scientific Ltd., UK) using Al $\mathrm{K} \alpha$ source. The specific Brunauer-Emmett-Teller (BET) surface areas were measured on a Quantachrome Quadrasorb Station 1 by nitrogen adsorption at $77.3 \mathrm{~K}$ and the pore-size distributions was measured on a Quantachrome Quadrasorb Station 1 by Barrett-Joyner-Halenda (BJH) pore size desorption isotherms at $77.3 \mathrm{~K}$ (sample weight: $0.1041 \mathrm{~g}$ and analysis time: $490.2 \mathrm{~min}$ ).

\section{Adsorption experiment}

The standard solutions of $\mathrm{Cr}(\mathrm{vI})$ with different concentrations (1.5 and $4.0 \mathrm{mg} \mathrm{L}^{-1}$ ) were obtained by dissolving potassium dichromate in distilled water. $\mathrm{Cr}(\mathrm{vI})$ solutions were treated with $1.0 \mathrm{~g} \mathrm{~L}^{-1}$ MCS to study the removal capacity. Briefly, the mixture was placed in an incubator shaker (100 rpm) at room temperature for $5 \mathrm{~min}$. Then, the MCS was removed gently with forceps. The clear solutions were collected and subjected to colorimetric analysis to determine the remaining $\mathrm{Cr}(\mathrm{vI})$ concentration. The $\mathrm{pH}$ study was conducted at different $\mathrm{pH}$ values from 2 to 11, the $\mathrm{pH}$ value was adjusted by using hydrochloric acid (using $1 \mathrm{~mol} \mathrm{~L}^{-1} \mathrm{HCl}$ to adjust $\mathrm{pH}$ from $\mathrm{pH} 2-$ 4 and concentrated $\mathrm{HCl}$ for $\mathrm{pH}=1$ ) or sodium hydroxide solutions $\left(1 \mathrm{~mol} \mathrm{~L}^{-1}\right)$. For colorimetric analysis, the aforementioned clear solutions $(5.25 \mathrm{~mL})$ were taken into test tubes, then $o$-phosphoric acid $(0.50 \mathrm{~mL}, 4.5 \mathrm{M})$ and DPC $\left(0.25 \mathrm{~mL}, 5 \mathrm{~g} \mathrm{~L}^{-1}\right)$ were added. After incubation at room temperature for 30 minutes for color development, the absorbance of the samples was measured in a UV-Vis spectrophotometer (Techcomp UV2310II) at $\lambda_{\max }=540 \mathrm{~nm}$ (Fig. S1a-f $\dagger$ ). To evaluate the efficiency of different adsorbents, removal percentage (RP, \%) is introduced as a criterion, which can be calculated using eqn (1):

$$
\mathrm{RP}=\frac{C_{0}-C_{\mathrm{r}}}{C_{\mathrm{r}}} \times 100 \%
$$

where $C_{0}$ is the initial concentration of the $\mathrm{Cr}(\mathrm{vI})$ solution and $C_{\mathrm{r}}$ represents the remaining $\mathrm{Cr}(\mathrm{vI})$ concentration after treatment. The adsorption capacity ( $q_{\mathrm{e}}, m$ of $\mathrm{Cr}(\mathrm{vI})$ per $\mathrm{g}$ of adsorption) of $\mathrm{Cr}(\mathrm{vI})$ was calculated by the following equation eqn (2):

$$
q_{\mathrm{e}}=\frac{\left(C_{0}-C_{\mathrm{e}}\right) \times V}{m}
$$


where $C_{0}\left(\mathrm{mg} \mathrm{L}^{-1}\right)$ and $C_{\mathrm{e}}\left(\mathrm{mg} \mathrm{L}^{-1}\right)$ are the initial and the equilibrium concentrations of dye. $V(\mathrm{~L})$ is the volume of aqueous solution, and $m(\mathrm{~g})$ is the weight of the adsorbents.

\section{Desorption experiment}

After adsorption experiment, the MCS was put into a conical flask containing $50 \mathrm{~mL} \mathrm{NaOH} \mathrm{(15} \mathrm{wt \% )} \mathrm{and} 50 \mathrm{~mL} \mathrm{NaCl}$ (15 wt\%). Then, the conical flask were stored in shaking incubator $(100 \mathrm{rpm})$ at $25{ }^{\circ} \mathrm{C}$ for $24 \mathrm{~h}$, and then taken out the MCS from the conical flask and dried it to be reused. At last, the waste liquid from the MCS after adsorption treatment was collected and sorted for centralized treatment.

\section{Results and discussion}

\section{Microstructure investigation of MCS}

Fig. 2a shown the scanning electron microscope (SEM) microstructure of the PP-C after microwave-assisted heating/ annealing treatments. The PP-C exhibited a sponge-like structure with homogeneous pores. The pores arranged sparsely, and the pore diameter was mostly centered at hundreds of nanometers. In contrast, the PPM-C exhibited a sponge-like structure with 3D interconnected pores by microscopic observation (Fig. 2b). Taking a closer view on the inner surfaces of the pores in PPM-C, the inset of Fig. $2 \mathrm{~b}$, it is interesting to observe a "shiatsu plates" structure decorated with ball-like protuberance and that is uniform distribution. We attribute the 3D interconnected pores to the microwaves selective heating: the reactants (polymers and MCC) with different dielectric constants led to the asynchronism of microwave power absorption value (Table $\mathrm{S} 2 \uparrow$ ), ${ }^{36,37}$ thus resulting in different direction of the steam migration in the transition state. Similarly, the $3 \mathrm{D}$ interconnected mesopores also derive from the channels remained of the steam migration. Their macro/micro scale structures provide a high surface area, a high surface-tobulk ratio, and in combination these features are suitable for the removal of heavy metal ions from water.

\section{Chemical structure of MCS}

The Fourier transform infrared (FT-IR) spectra of MCC, PVA, PVP and as-prepared PPM-C were presented in Fig. 3a. The basic characteristic peaks of MCC appeared at $3334 \mathrm{~cm}^{-1}(\mathrm{O}-\mathrm{H}$
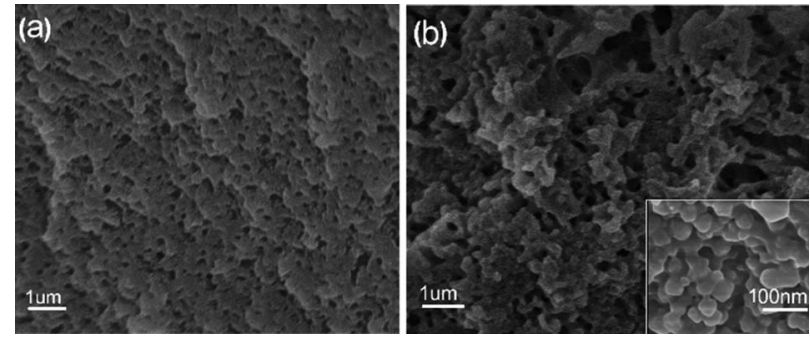

Fig. 2 SEM images of (a) PP-C and (b) PPM-C (PP-C and PPM-C represent the composition of the carbon sponge containing PVA/PVP and PVA/PVP/MCC, respectively).
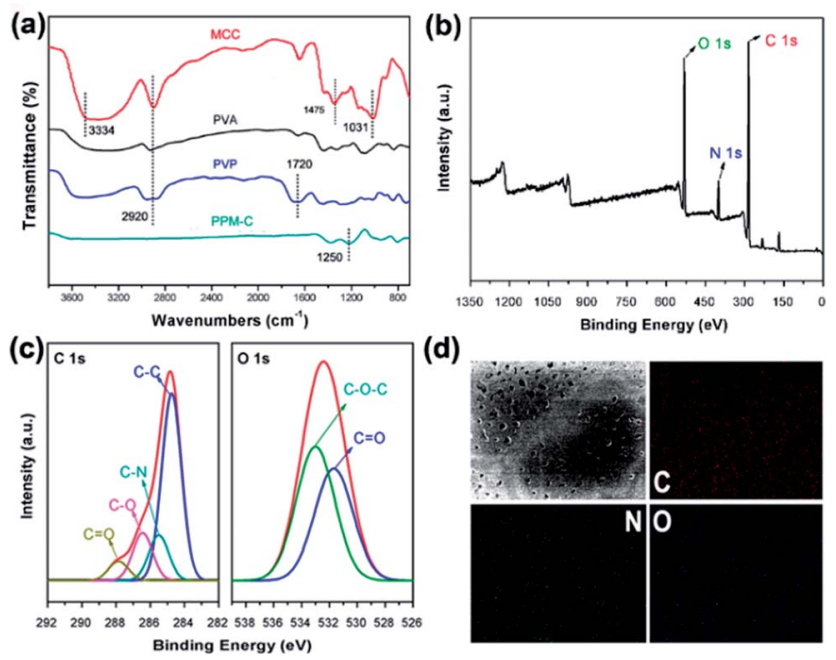

(d)



Fig. 3 (a) FT-IR spectra of the MCC, PVA, PVP, and PPM-C, (b) the whole survey XPS spectrum of the PPM-C, (c) $C$ 1s and $O$ 1s XPS spectrum of the PPM-C, (d) energy dispersive $X$-ray images with the corresponding mapping of $\mathrm{C}, \mathrm{N}$, and $\mathrm{O}$ elements.

stretching vibration), $2920 \mathrm{~cm}^{-1}$ (C-H stretching vibration), $1475 \mathrm{~cm}^{-1}$ (C-H framework vibration) and $1031 \mathrm{~cm}^{-1}$ (C-O stretching vibration). The basic characteristic peaks of PVP appeared at $1720 \mathrm{~cm}^{-1}(\mathrm{C}=\mathrm{O}$ stretching vibration of the pyrrole ring). Comparing the FT-IR spectra of MCC, PVA, PVP, and PPM$\mathrm{C}$, the spectrum ranged from 4000 to $1700 \mathrm{~cm}^{-1}$ without stretching vibration peaks in PPM-C, which explained that the chains of MCC, PVA and PVP were degraded to carbon skeleton through the microwave-assisted annealing process. The characteristic absorption band around $1350 \mathrm{~cm}^{-1}$ could be assigned to the absorption peak of the stretching vibration of ether bond $(\mathrm{O}-\mathrm{C}-\mathrm{O}) .^{38}$ The ether bond derives from the ketalization reaction between ketones of PVP $(\mathrm{PVP}-\mathrm{C}=\mathrm{O})$ and alcoholic hydroxyl groups of PVA (PVA-OH) (Fig. S2 $\dagger$ ). ${ }^{39}$ And X-ray photoelectron spectroscopy (XPS) measurement was carried out to further investigate functional groups presented on the surface of asprepared PPM-C. The whole XPS survey spectrum of the asprepared PPM-C (Fig. 3b) exhibited three obvious peaks at $284.84,399.33$, and $532.3 \mathrm{eV}$, which were attributed to C $1 \mathrm{~s}, \mathrm{~N} 1 \mathrm{~s}$ and $\mathrm{O} 1 \mathrm{~s}$, respectively. The $\mathrm{C} 1 \mathrm{~s}$ XPS spectrum (Fig. 3c) can be further deconvoluted into three carbon states at 284.84, 285.40, 286.15 and $288.7 \mathrm{eV}$, which were attributed to $\mathrm{C}-\mathrm{C}, \mathrm{C}-\mathrm{N}, \mathrm{C}-\mathrm{O}$ and $\mathrm{C}=\mathrm{O}$ respectively. ${ }^{40}$ Moreover, the peak of double bond $(\mathrm{C}=\mathrm{O})$ near $289 \mathrm{eV}$ disappeared, thus further verifying the ketalization reaction between ketones of PVP and alcoholic hydroxyl groups of PVA. The O 1s spectrum (Fig. 3c) showed two peaks at 533.2 and $531.6 \mathrm{eV}$, which was assigned to $\mathrm{O}-\mathrm{C}-\mathrm{O}$ and $\mathrm{C}=\mathrm{O}$ bands. The $\mathrm{C}-\mathrm{O}-\mathrm{C}$ peak indicated the successful ketalization reaction between ketones of PVP and alcoholic hydroxyl groups of PVA, which was further confirmed by the energy dispersive X-ray spectra (EDS) images with the corresponding element mappings in Fig. 3d. These results showed that the presence of these key functional groups $(\mathrm{C}-\mathrm{C}, \mathrm{C}-\mathrm{N}$, and $\mathrm{C}-\mathrm{O})$ on the surface of the as-prepared PPM-C without being broken, ${ }^{41}$ 
indicating that the carbon skeleton morphology was well maintained after the annealing process. Namely, the precursors were initially heated by the microwave-assisted heating, in which the PVP chain was linked to PVA chain by ether bonds (O$\mathrm{C}-\mathrm{O}$ ). Meanwhile, MCC as a filling agent was bound together with the polymer network by hydrogen bonds, obtaining a sollike solution. Then, the sol-like solution was annealed to obtain carbon sponge (PPM-C) with the ordered carbon skeleton. The schematic formation mechanism of the PPM-C was also illustrated in Fig. 1.

\section{The interfacial behavior of MCS}

Specific surface area and pore size are critically important for mesoporous carbon materials as adsorbents for the removal of heavy metal ions from water. $\mathrm{N}_{2}$ physisorption isotherms were applied to evaluate the surface properties of the carbon sponge. In order to determine specific surface area and pore size, a linear standard curve relating has been made (Fig. 4a). As can be seen in Fig. $4 \mathrm{~b}, \mathrm{~N}_{2}$ sorption isotherms of the PPM-C are the representative type $\mathrm{V}$ reversible sorption (the curve in the inset), indicative of a typical mesoporous network structure. ${ }^{42}$ This is confirmed by the Barrett-Joyner-Halenda (BJH) pore size distribution curves calculated from the desorption branches (Fig. 4c). It is clearly shown that the pore radius size of PPM-C was highly centered at $\sim 25 \AA$ compared with PP-C at $\sim 70 \AA$, and their characteristic diameter of $\sim 50 \AA$ was in the mesoporous range of $20-500 \AA{ }^{41}$ verifying the carbon sponge with 3D interconnected mesoporous structures. Moreover, the PPM-C exhibited the high specific surface area of $310.10 \mathrm{~m}^{2}$ $\mathrm{g}^{-1}$, which is much higher than $99.73 \mathrm{~m}^{2} \mathrm{~g}^{-1}$ for PP-C. One of the major reasons for the increased specific surface area of the PPM-C is that the mismatch between MCC and their surrounding polymer matrix induced the surface elastic

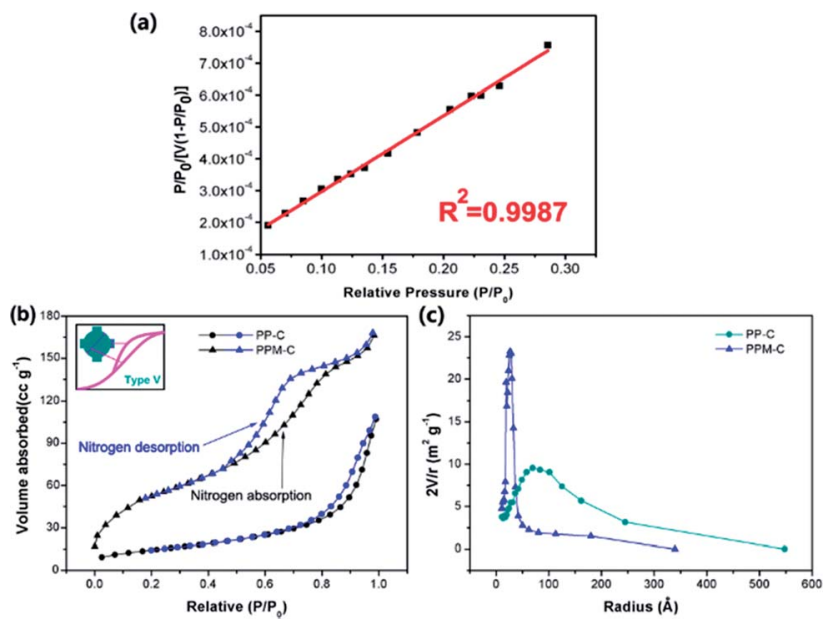

Fig. 4 (a) Linear relationship between the relative pressure and adsorption, (b) nitrogen absorption-desorption isotherms curves of PP-C (circle) and PPM-C (up triangle) calculated using the BET method (black: nitrogen absorption curve, blue: nitrogen desorption curve, inset: the type $V$ of gas adsorption isotherms), (c) the pore-size distribution of PP-C (circle) and PPM-C (up triangle) calculated using the $\mathrm{BJH}$ method. instability, which lead to the ball-like protuberances in the process of cooling for increasing the surface area per unit weight. Importantly, the adsorption-desorption curves of PPM-C exhibited discernible steps over the entire relative pressure range (Fig. 4b). This result may be interpreted that the adsorption process is initially similar to that on macroporous solids, but at higher pressures the amount adsorbed rises steeply due to the capillary condensation in mesopores. ${ }^{43,44}$ The amount adsorbed rises steeply of PPM-C from the relative pressure value at 0.6 , compared with that of PP-C at 0.9 , verifying that the capillary condensation of PPM-C in mesopores is particularly prominent. Moreover, the adsorption isotherms for PPM-C exhibited high-pressure hysteresis loops (with a closed hysteresis loop), indicating the adsorbent without swelling and chemisorption during the adsorption process. Consequently, the 3D interconnected mesopores and an evenly distributed ball-like protuberance on the surface enable the PPM-C to achieve high specific surface area and multilevel structures for the purification of wastewater.

\section{Cr(vi) removal by MCS}

The obtained PP-C and PPM-C were used as adsorbents to remove $\mathrm{Cr}(\mathrm{VI})$ from pre-prepared $\mathrm{Cr}(\mathrm{VI})$ aqueous solution. As depicted in the Fig. 5, the adsorption mechanisms of PP-C and PPM-C for Cr(vI) are different. The Cr(vI) on PP-C with a smooth interface is a single-layer adsorption just like active carbon benefited from high specific surface area. In contrast, the high adsorption capacity of $\mathrm{Cr}(\mathrm{VI})$ on PPM-C benefits from the synergistic effect of the increased specific surface area and multilevel structures that consist of 3D interconnected mesopores and a rough interface decorated with ball-like protuberance.

In order to determine the change of trace concentrations of $\mathrm{Cr}(\mathrm{vI})$, a linear standard curve relating the UV-Vis peak intensity at $540 \mathrm{~nm}$ has been made (Fig. 6a). The residual $\mathrm{Cr}(\mathrm{vI})$ concentration after treatment can be calculated from the standard curve. Fig. S4a and c $\dagger$ show the UV-Vis absorption of the $1.5 \mathrm{mg} \mathrm{L}^{-1}$ and $4.0 \mathrm{mg} \mathrm{L}^{-1} \mathrm{Cr}(\mathrm{vI})$ solution and the solutions after treatment with PP-C and PPM-C in an incubator shaker for 5 min, which are consistent with the standard curve (Fig. 6a). After treated with different PP-C and PPM-C in an incubator shaker for $5 \mathrm{~min}$, the peak intensity decreased gradually with increasing the concentration of PVP and MCC. Moreover, the peak intensity after treated with carbon sponge with MCC
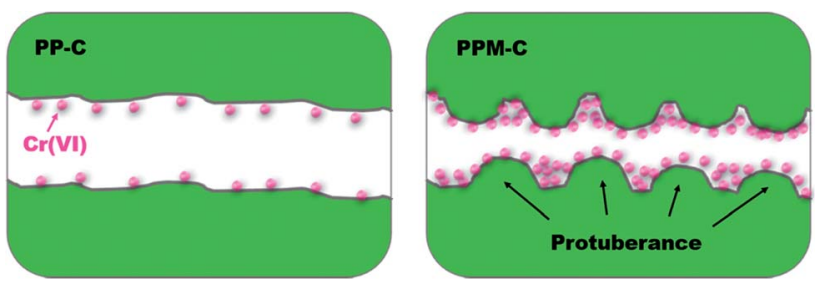

Fig. 5 Schematic illustration of carbon sponge for $\mathrm{Cr}(\mathrm{VI})$ removal (PP$C$ with a smooth interface and PPM- $C$ with a rough interface decorated with ball-like protuberance) (cross-sectional view). 

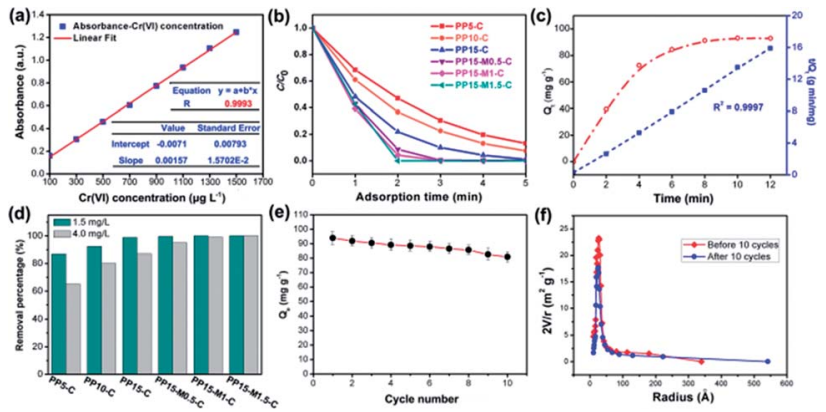

Fig. 6 (a) Linear relationship between the $\mathrm{Cr}(\mathrm{VI})$ concentration and UV-Vis absorption tested at room temperature. (b) The Cr(vI) removal performance of different carbon sponge (adsorbent: $1.0 \mathrm{~g} \mathrm{~L}^{-1}, \mathrm{Cr}(\mathrm{VI})$ : $1.5 \mathrm{mg} \mathrm{L}^{-1}$, where $C_{0}$ represents the initial concentration of the $\mathrm{Cr}(\mathrm{vI})$ and $C$ represents the concentration of the $\mathrm{Cr}(\mathrm{vI})$ after adsorption), and (c) kinetic adsorption data plots of $\mathrm{Cr}(\mathrm{VI})$ adsorption by PPM-C: $\mathrm{Cr}(\mathrm{vI})$ removal rate $q_{t} v s$. time (open circle) and the transformed rate plot $t / q_{t}$ $v s$. time (solid square). (d) The RP of different carbon sponge vs. time at different initial concentrations of $\mathrm{Cr}(\mathrm{VI})$ (adsorbent: $1.0 \mathrm{~g} \mathrm{~L}^{-1}$ ), (e) adsorption capacities of PP15-M1.5-C as a function of repeated adsorption-desorption cycles for the $\mathrm{Cr}(\mathrm{vI})$ removal. (f) The changes of pore-size distribution of the PP15-M1.5-C before and after 10 adsorption-desorption cycles.

decreased significantly, compared with that without MCC. Upon the subsequent EDS measurement, Fig. S3a-f and $\mathrm{g}-\mathrm{l} \dagger$ exhibit the composition of PPM-C before and after $\operatorname{Cr}(\mathrm{vI})$ removal, respectively. One can observe that the $\mathrm{Cr}$ map (Fig. S31 $\dagger$ ) of the PPM-C after $\mathrm{Cr}(\mathrm{vI})$ removal appeared chromium fluorescent points, verifying PPM-C for $\mathrm{Cr}(\mathrm{vI})$ removal from another side. The $\mathrm{Cr}(\mathrm{vI})$ removal percentage treated by different PP-C and PPM-C is shown in Fig. 6b. It can be found that the $\mathrm{Cr}(\mathrm{vI}) \mathrm{RP}$ of PPM-C with different contents of MCC are all close to $100 \%$ within 5 min while the highest $\mathrm{Cr}(\mathrm{vI}) \mathrm{RP}$ of PP-C barely reach to $92 \%$. With a very short treatment time of $5 \mathrm{~min}$, the removal capacity of PPM-C reaches $93.96 \mathrm{mg} \mathrm{g}^{-1}$ by physical adsorption, which is much higher than those of previously reported other materials (Table S2 $\dagger$ ). Furthermore, the PPM-C exhibits gradually stronger adsorption properties with increasing the MCC content, indicating that MCC as a filling agent is critical for highly efficient $\mathrm{Cr}(\mathrm{vI})$ removal. Therefore, PPM-C will be used as the model compound to systematically study the $\mathrm{Cr}(\mathrm{vI})$ removal kinetics and mechanisms. The kinetics of the adsorption describing the $\mathrm{Cr}(\mathrm{vI})$ uptake rate is one of the important characteristics which control the residence time of adsorbate uptake at the solid-liquid interface. Hence, in the present study, the kinetics of $\mathrm{Cr}(\mathrm{vI})$ removal was carried out to understand the adsorption behavior of the as-prepared PPM-C. Fig. 6c shows the $\mathrm{Cr}(\mathrm{vI})$ adsorption data over PPM-C at different time intervals (open circle). By evaluate the suitability of different models including pseudo-first-order ${ }^{45}$ pseudo-secondorder ${ }^{46}$ Elovich $^{47}$ and intraparticle diffusion, ${ }^{48}$ the kinetics of $\mathrm{Cr}(\mathrm{vI})$ removal for the pseudo-second-order model was the highest correlation coefficient of $R^{2}=0.9997$ (Fig. 6c, square symbol curve), indicating that pseudo-second-order model provides an excellent correlation for the adsorption of $\mathrm{Cr}(\mathrm{vI})$ on PPM-C.
The pseudo-second-order model equation is given as eqn (3):

$$
\frac{t}{Q_{t}}=\frac{1}{k_{\mathrm{ad}} Q_{\mathrm{e}}^{2}}+\frac{t}{Q_{\mathrm{e}}}
$$

where $Q_{t}$ is the solid-phase loading of $\mathrm{Cr}(\mathrm{vI})$ in the adsorbent at time $t, Q_{\mathrm{e}}$ is the adsorption capacity at equilibrium, and $k_{\mathrm{ad}}$ is the rate constant of adsorption.

Fig. 6d shown that the RP of PPM-C with different components all reached to $\sim 100 \%$ at a low concentration of $\operatorname{Cr}(\mathrm{vI})$ (1.5 mg L ${ }^{-1}$ ), while the RP of PP-C increased from $86.91 \%$ to $98.87 \%$ upon an increase of concentration of PVP. To further investigate the performance of $\mathrm{Cr}(\mathrm{vI})$ removal in a high concentration conditions, PP-C and PPM-C were tested in a $\mathrm{Cr}(\mathrm{VI})$ solution of $4.0 \mathrm{mg} \mathrm{L}^{-1}$. It is obvious that the RP of PP-C decreased than that of $1.5 \mathrm{mg} \mathrm{L}^{-1}(65.32-87.54 \%)$. By comparison, the RP of PPM-C with a slight decrease further displayed the superior performance of PPM-C for $\mathrm{Cr}(\mathrm{vI})$ removal compared with PP-C. To further investigate the recyclability of the MCS, recycled adsorption-desorption experiments were carry out. After 10 cycles, the adsorption capacity of the MCS still maintained $\sim 86 \%$, indicating that the MCS can be regenerated and reused, which contribute to the cost savings in a large scale (Fig. 6e). Fig. $6 \mathrm{f}$ demonstrates the changes of pore-size distribution of the MCS before and after 10 adsorption-desorption cycles. After 10 cycles, the pore-area of the mesopores in the characteristic diameter of $\sim 50 \AA$ had a certain decrease, compared with the initial state of the MCS, due to that the tiny amounts of $\mathrm{Cr}(\mathrm{vr})$ attached to the surface and then lead to the clogging of mesopores. Fig. 7a-e show the process of highly efficient $\mathrm{Cr}(\mathrm{vI})$ removal visually (Video $\mathrm{S} 1 \dagger$ ) and the solution after $\mathrm{Cr}(\mathrm{vI})$ removal could be applied directly for water culture experiment (Fig. 7g-i). Moreover, the PPM-C maintained the stability structure after $\mathrm{Cr}(\mathrm{vI})$ removal so that the adsorbent was removed from the wastewater without causing second-pollution (the inset of Fig. 7f).

\section{$\mathrm{Cr}(\mathrm{vI})$ removal percentage at different conditions}

Fig. 8a displayed the faster $\mathrm{Cr}(\mathrm{vI})$ removal of PPM-C under dynamic adsorption compared with the PPM-C under static adsorption, indicating that the mobility of the water contributes to highly efficient $\mathrm{Cr}(\mathrm{vI})$ removal. It was found that the

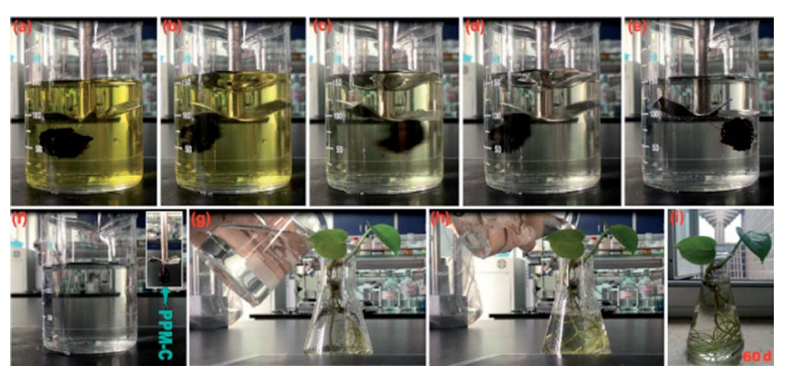

Fig. 7 (a)-(e) Color changes of the $\mathrm{Cr}(\mathrm{VI})$ solutions during the adsorption process by the PPM-C, and (f) the PPM-C can removed from the water with morphological integrity. (g) and (h) The $\mathrm{Cr}(\mathrm{vI})$ solution after treatment by PPM-C be used for water culture experiment, and (i) the plant grown well after 60 days. 

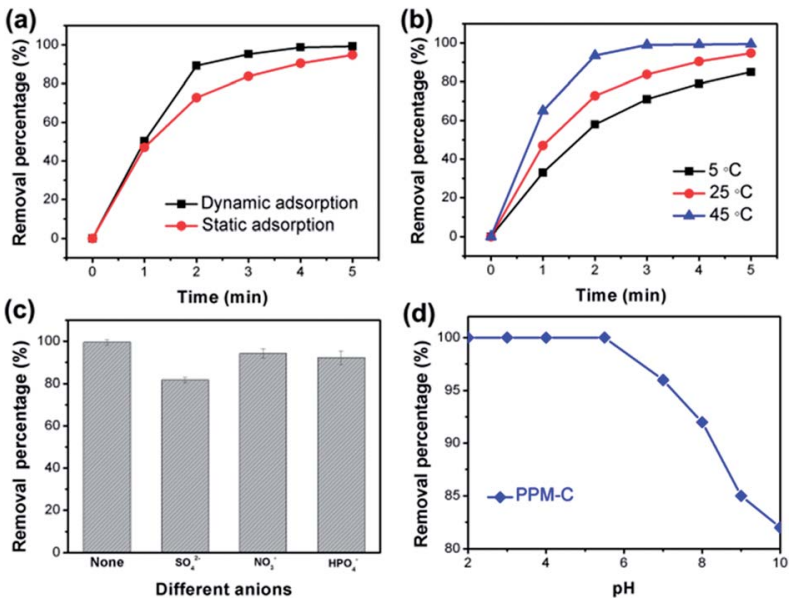

Fig. 8 (a) The $\mathrm{Cr}(\mathrm{VI}) \mathrm{RP}$ of PPM-C vs. time under dynamic adsorption $(100 \mathrm{rpm})$ and static adsorption at room temperature $\left(25^{\circ} \mathrm{C}\right)$, (b) the $\mathrm{Cr}(\mathrm{VI}) \mathrm{RP}$ of PPM-C vs. time at different temperature (under static adsorption), (c) effect of competing anions, and (d) solution $\mathrm{pH}$ on the $\mathrm{Cr}(\mathrm{VI}) \mathrm{RP}$ of PPM-C (the concentration of $\mathrm{SO}_{4}{ }^{2-}\left(\mathrm{Na}_{2} \mathrm{SO}_{4}\right), \mathrm{NO}_{3}{ }^{-}$ $\left(\mathrm{NaNO}_{3}\right)$, and $\mathrm{H}_{2} \mathrm{PO}_{4}^{-}\left(\mathrm{KH}_{2} \mathrm{PO}_{4}\right)$ is $\left.0.01 \mathrm{M}\right)$. (PPM-C for the $\mathrm{Cr}(\mathrm{VI})$ removal at different conditions is PP15-M1.5-C).

temperature is also one of the most important variables affecting the $\mathrm{Cr}(\mathrm{vI})$ removal efficiency. As displayed in Fig. 8b, the RP of PPM-C reached to $\sim 100 \%$ at $45^{\circ} \mathrm{C}$, while the RP barely increased to $86.7 \%$ at $5{ }^{\circ} \mathrm{C}$. Therefore, the high temperature had positive influence on the $\mathrm{Cr}(\mathrm{vr})$ removal process, ascribing to the promoting effect of the higher temperature for efficient ions transfer. Besides, different anions in the wastewater can affect the $\mathrm{Cr}(\mathrm{vI})$ removal. The effect of anions in different valences (divalent and monovalent) on $\mathrm{Cr}(\mathrm{vI})$ removal was shown in Fig. 8c. The inhibition of the divalent anions $\left(\mathrm{SO}_{4}{ }^{2-}\right)$ on the $\mathrm{Cr}(\mathrm{vI})$ removal is much more serious than that of the monovalent anions $\left(\mathrm{NO}_{3}{ }^{-}\right.$and $\left.\mathrm{H}_{2} \mathrm{PO}_{4}{ }^{-}\right)$, which could be explained by the divalent anions with a higher ion charge density compared with monovalent anions, ${ }^{49}$ making a better affinity of the divalent anions toward adsorbent. To further investigate the influence of solution $\mathrm{pH}$ for the adsorption characteristics, the $\mathrm{Cr}(\mathrm{vI})$ RP of the PPM-C in solutions of different pH is shown in Fig. 8d. One can observe that the complete $\mathrm{Cr}(\mathrm{vI})$ removal was achieved under acidic conditions when the $\mathrm{pH}$ was controlled at 2, 3, 4, and 5.5 within $5 \mathrm{~min}$. Increasing the solution $\mathrm{pH}$ to 8 and even higher, the $\mathrm{Cr}(\mathrm{VI}) \mathrm{RP}$ sharply decreased to $81.72 \%$. This result may be interpreted that the large amount of $\mathrm{OH}^{-}$ions under alkaline conditions compete with $\mathrm{Cr}(\mathrm{vI})$ species on the MCS surface, leading to the decline of $\mathrm{Cr}(\mathrm{VI}) \mathrm{RP}$.

\section{Conclusions}

In summary, we have manufactured a MCS from one-pot synthesis method of a supramolecular microcrystalline cellulose-polymer system by microwave-assisted heating/annealing treatments. The as-prepared products exhibited high adsorption capacity of $93.96 \mathrm{mg} \mathrm{g}^{-1}$ and fast $\mathrm{Cr}(\mathrm{vr})$ removal from wastewater only within $5 \mathrm{~min}$. Our studies suggest that the key to obtain a very large removal capacity of $\operatorname{Cr}(\mathrm{vI})$ and a fast removal rate is the synergistic effect of the high specific surface area and multilevel structures that consist of 3D interconnected mesopores and a rough interface decorated with ball-like protuberance. More significantly, the carbon sponge are recyclable without causing second-pollution, and thus has great potential applications in environmental and water purification fields.

\section{Conflicts of interest}

There are no conflicts to declare.

\section{Acknowledgements}

This work was supported by the Fundamental Research Funds for the Central Universities (No. 2017ZY49) and the Foundation (No. KF201607) of Key Laboratory of Pulp and Paper Science and Technology of Ministry of Education/Shandong Province of China.

\section{References}

1 L. C. Hsu, S. L. Wang, Y. C. Lin, M. K. Wang, P. N. Chiang and J. C. Liu, Environ. Sci. Technol., 2010, 44, 6202-6208.

2 M. Owlad, M. K. Aroua, W. A. W. Daud and S. Baroutian, Water, Air, Soil Pollut., 2009, 200, 59-77.

3 L. Monser and N. Adhoum, Sep. Purif. Technol., 2002, 26, 137-146.

4 N. Kongsricharoern and C. Polprasert, Water Sci. Technol., 1996, 34, 109-116.

5 S. Luo, F. Qin, Y. Ming, H. Zhao, Y. Liu and R. Chen, J. Hazard. Mater., 2017, 340, 253-262.

6 R. Wang, G. Cheng, Z. Dai, J. Ding, Y. Liu and R. Chen, Chem. Eng. J., 2017, 327, 371-386.

7 G. Li, F. Qin, R. Wang, S. Xiao, H. Sun and R. Chen, J. Colloid Interface Sci., 2013, 409, 43-51.

8 J. R. Werber, C. O. Osuji and M. Elimelech, Nat. Rev. Mater., 2016, 1, 16018.

9 S. Bolisetty and R. Mezzenga, Nat. Nanotechnol., 2016, 11, 365-373.

10 J. H. Zhu, S. Y. Wei, H. B. Gu, S. B. Rapole, Q. Wang, Z. P. Luo, N. Haldolaarachchige, D. P. Young and Z. H. Guo, Environ. Sci. Technol., 2012, 46, 977-985.

11 E.-T. Liu, H. Zhao, H. Li, G. Li, Y. Liu and R. Chen, New J. Chem., 2014, 38, 2911-2916.

12 F. Qin, G. Li, H. Xiao, Z. Lu, H. Sun and R. Chen, Dalton Trans., 2012, 41, 11263-11266.

13 J. H. Zhu, H. B. Gu, J. Guo, M. J. Chen, H. G. Wei and Z. P. Luo, J. Mater. Chem. A, 2014, 2, 2256-2265.

14 Q. Huang, M. Y. Liu, R. Guo, L. C. Mao, Q. Wan, G. J. Zeng, H. Y. Huang, F. J. Deng, X. Y. Zhang and Y. Wei, J. Mater. Sci., 2016, 51, 9625-9637.

15 M. Noked, E. Avraham, A. Soffer and D. Aurbachand, J. Phys. Chem. C, 2009, 113, 21319-21327.

16 J. Liang, Y. Huang, L. Zhang, Y. Wang, Y. Ma and T. Guo, Adv. Funct. Mater., 2009, 19, 2297-2302. 
17 Q. Yang, F. Lan, Q. Y. Yi, Y. Wu and Z. W. Gu, Nanoscale, 2015, 7, 17617-17622.

18 Z. X. Wang, J. Guo, J. Ma and L. Shao, J. Mater. Chem. A, 2015, 3, 19960-19968.

19 Y. Z. Zhu, X. C. Li, G. H. He and X. H. Qi, Chem. Commun., 2015, 51, 2991-2994.

20 H. Y. Ma, B. S. Hsiao and B. Chu, ACS Macro Lett., 2012, 1, 213-216.

21 N. Du, H. Zhang, B. D. Chen, X. Y. Ma, Z. H. Liu and J. B. Wu, Adv. Mater., 2007, 19, 1641-1645.

22 X. Gui, Z. Zeng, Z. Lin, Q. Gan, R. Xiang, Y. Zhu, A. Cao and Z. Tang, ACS Appl. Mater. Interfaces, 2013, 5, 5845-5850.

23 C. Su, H. Yang, S. Song, B. Lu and R. Chen, Chem. Eng. J., 2017, 309, 366-373.

24 C. Su, H. Yang, H. Zhao, Y. Liu and R. Chen, Chem. Eng. J., 2017, 330, 423-432.

25 S. M. Alatalo, F. Pileidis, E. Makila, M. Sevilla, E. Repo and J. Salonen, ACS Appl. Mater. Interfaces, 2015, 7, 25875-25883.

26 J. H. Li, J. Y. Li, H. Meng, S. Y. Xie, B. W. Zhang and L. F. Li, J. Mater. Chem. A, 2014, 2, 2934-2941.

$27 \mathrm{H}$. Hu, Z. B. Zhao, W. B. Wan, Y. Gogotsi and J. S. Qiu, Adv. Mater., 2013, 25, 2219-2223.

28 H. Y. Sun, Z. Xu and C. Gao, Adv. Mater., 2013, 25, 2554-2560.

29 X. Yang, K. Y. Shi, I. Zhitomirsky and E. D. Cranston, Adv. Mater., 2015, 27, 6104-6109.

30 E. S. Steigerwalt, G. A. Deluga and C. M. Lukehart, J. Phys. Chem. B, 2002, 106, 760-766.

31 J. Yan, H. Zhou, P. Yu, L. Su and L. Mao, Electrochem. Commun., 2008, 10, 761-765.

32 Y. F. Zhao, B. Li, Q. Wang, W. Gao, C. L. J. Wang and M. Wei, Chem. Sci., 2014, 5, 951-958.

33 K. H. Lee, Y. W. Lee, S. W. Lee, J. S. Ha, S. S. Lee and J. G. Son, Sci. Rep., 2010, 25, 13696-13706.
34 J. A. Gerbec, D. Magana, A. Washington and G. F. Strouse, J. Am. Chem. Soc., 2005, 127, 15791-15800.

35 B. Hu, S. B. Wang, K. Wang, M. Zhang and S. H. Yu, J. Phys. Chem. C, 2008, 112, 11169-11174.

36 B. C. Luo, X. H. Wang, Y. P. Wang and L. T. Li, J. Mater. Chem. A, 2014, 2, 510-519.

37 S. Takechi, Y. Teramoto and Y. Nishio, Cellulose, 2016, 23, 765-777.

38 L. Gao, J. G. Han and X. G. Lei, Org. Lett., 2016, 18, 360-363. 39 Y.-J. Liu, L.-H. Fu, S. Liu, L.-Y. Meng, Y.-Y. Li and M.-G. Ma, J. Mater. Chem. B, 2016, 4, 4847-24854.

40 S. Sahu, B. Behera, T. K. Maiti and S. Mohapatra, Chem. Commun., 2012, 48, 8835-8837.

41 R. Kumar, D. Kumar and A. K. Chakraborti, Synthesis, 2007, 2, 299-303.

42 M. Kruk and M. Jaroniec, Chem. Mater., 2001, 13, 3169-3183. 43 K. S. W. Sing, D. H. Everett, R. A. W. Haul, L. Moscou, R. A. Pierotti, J. Rouquerol, T. Siemieniewska, K. S. W. Sing, D. H. Everett, R. A. W. Haul, R. A. Pierotti, R. A. W. L. Mouscou, L. Moscow and T. Siemienewska, Pure Appl. Chem., 1985, 57, 603-619.

44 Y. S. Ho, G. McKay, D. A. J. Wase and C. F. Forster, Adsorpt. Sci. Technol., 2000, 18, 639-650.

45 S. Lagergren, Handlingar, 1898, 24, 1-39.

46 Y. S. Ho, G. McKay, D. A. J. Wase and C. F. Forster, Adsorpt. Sci. Technol., 2ss000, 18, 639-650.

47 R. S. Juang and M. L. Chen, Ind. Eng. Chem. Res., 1997, 36, 813-820.

48 S. K. Srivastava, R. Tyagi and N. Pant, Water Res., 1989, 23, 1161-1165.

49 J. Zhao, X. Zhang, X. He, M. Xiao, W. Zhang and C. Lu, J. Mater. Chem. A, 2015, 3, 14703-14711. 